\title{
Magnetic nanoparticles: novel options for vascular repair?
}

\author{
“...MNPs can be utilized as carriers for drug, gene or \\ cell therapy, reducing the required dose or amount and \\ limiting adverse side effects."
}

First draft submitted: 29 January 2016; Accepted for publication: 8 February 2015; Published online: 16 March 2016

To date nanotechnology offers great promise for life sciences and healthcare. Nanoparticles with their small dimensions, high volume to surface ratio and special physicochemical properties enable the development of novel diagnostic and therapeutic procedures. For biomedical applications, superparamagnetic nanoparticles are preferentially used. They display strong magnetic properties when exposed to a magnetic field and show no residual magnetization in its absence, which reduces their aggregation. A frequently used type of magnetic nanoparticles (MNPs) is that of the core shell type [1,2] comprising a magnetic core based on iron oxide and a polymer coating that stabilizes the particle. Iron oxide based MNPs are widely used because they display good biocompatibility. Moreover, the biological response to the MNPs is determined by their coating that also defines the functionality of the particle [3]. For example, the composition of the surface controls the adsorption of drugs, the complex formation with nucleic acids and viral vectors as well as the uptake of the MNPs by cells. Additionally, by the attachment of targeting ligands such as antibodies a tissue-specific delivery can be facilitated. Thus, MNPs can be utilized as carriers for drug, gene or cell therapy, reducing the required dose or amount and limiting adverse side effects. This is achieved due to active targeting by site-specific delivery of the load mostly using specifically designed magnetic fields. Additionally, MNPs can be applied as contrast agent for imaging purposes. This is then referred to as 'theranostics', the combination of therapy and diagnostics [4].
Blood vessels are ideal targets for MNPdependent treatment and also diagnostic approaches because MNPs can be directly injected into the vasculature. Consequently, they are transported by the bloodstream thereby potentially reaching all vascular beds within the body. Especially the endothelium is easy to target because it covers the inner surface of the vessel and therefore no penetration of MNPs through tissues is necessary. Although vascular disease is mostly systemic, there exist special predilection sites in vascular segments with perturbed blood flow (e.g., carotid bifurcation) that require a local therapy. Thus, with the help of MNPassisted drug, gene and cell therapy vascular disease of all stages can be treated.

Vascular disease can result in atherosclerosis and ischemic end organ damage presenting as heart attack or stroke and is the leading cause of death worldwide. Therefore, MNPs applied for vascular repair could help to alleviate a severe public health problem with a high economic burden. Current therapeutic strategies of vascular disease have several drawbacks. At the early stage, systemic drug applications are employed that reduce risk factors for vascular disease such as high cholesterol levels or elevated blood pressure but the drugs have a limited half-life, display no site specificity in the vasculature and do not directly act on the endothelium. At later stages when atherosclerotic plaques have already formed invasive procedures such as balloon angioplasty, stent implantation or surgical plaque removal are required. However, all these interventions damage the vas-

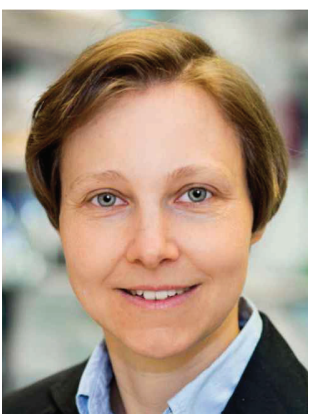

Daniela Wenzel

Institute of Physiology I, University Clinic Bonn, Bonn, Germany Tel.: +492286885216 Fax: +492286885201 dwenzel@uni-bonn.de 
cular wall, which can lead to restenosis or thrombosis. Thus, novel treatment options for vascular disease are urgently needed.

\section{Where we stand now: experimental vascular therapies using MNPs}

Drug therapy

Drug therapy of vascular disease has been proposed for the inhibition of angiogenesis in atherosclerotic plaques [5] and the prevention of restenosis after surgical intervention [6]. However when systemically administered, anti-angiogenic or cytostatic agents often display unwanted side effects. To enable a local application of the compounds they were adhered to balloons or stents [7] that are used for percutaneous transluminal angioplasty. This strategy and especially the establishment of drug-eluting stents loaded with anti-proliferative agents has strongly improved clinical outcome with restenosis rates below 10\% [8]. However, balloons and stents can only release pharmacological agents during a limited time span without the option to reload the drug. To improve the duration and local efficiency of drug release, a combination of MNPs and external magnetic fields is very promising. Paclitaxelloaded MNPs were employed after arterial ligation in a rat carotis stenting model. Thereby an external magnetic field strongly increased the deposition and slowed the elimination rate of drug-loaded MNPs after restoration of blood flow, which resulted in reduced instent restenosis [9]. However, also the use of stents has several disadvantages as it cannot be applied in special anatomical locations (e.g., vascular bifurcations) and it inhibits re-endothelialization resulting in instent thrombosis. In order to avoid stents, in another study MNPs were loaded with the anti-angiogenic drug fumagillin and site-specificity was provided by a peptide specific for vascular av $\beta 3$ integrins; this strategy resulted in an inhibition of plaque angiogenesis in atherosclerotic rabbits [10]. Similarly, rapamycinloaded av $\beta 3$-integrin-specific MNPs were locally applied to rabbit arteries after balloon injury leading to a reduction of restenosis [11]. In both studies, MNPs also enabled MRI analysis confirming the intramural binding of drug-loaded particles and permitting quantification of plaque neovascularization.

\section{Gene therapy}

To obtain a long-lasting therapeutic effect, gene therapy approaches may be better suited than drug-based strategies. For vascular treatment, different delivery vectors have been tested. While nonviral vectors demonstrated low efficiency, especially lentiviruses and adeno-associated viruses proved to be better suited for prolonged vascular expression [12]. For endothelial treat- ment intravascular application of the vectors appears most promising, however, the continuous blood flow impedes targeting and stop-flow conditions bear the risk of ischemia. This may be also an important reason for the lack of efficiency of gene therapy in clinical trials addressing vascular diseases so far. Up to now there are only very few studies aiming at an MNP-dependent localized gene transfer within vessels under blood flow. Chorny et al. could show that adenovirus-loaded MNPs enable vascular gene transfer under blood flow in a rat model of stent angioplasty [13]. Moreover, our group demonstrated that lentivirus/MNP complexes and external magnets can be used for the local transduction of the native endothelial cell layer in ex vivo perfused mouse aortas without stents. Additionally, we could illustrate that the placement of external magnets on the abdominal wall altered tissue distribution after intravascular injection of the complexes in mouse in vivo [14]. These studies, however, are somewhat limited because they only showed reporter gene expression in the vessels. So it is unclear if the experimental strategies are efficient enough to allow functional improvement when therapeutic genes are applied. Functional genes/proteins of special interest for endothelial overexpression may be eNOS that augments NO bioavailability and enhances vascular function at early stage vascular disease as well as angiogenic growth factors that induce collateral growth and perfusion of ischemic tissues in late stage occlusive disease.

\section{Endothelial cell therapy}

After interventional or surgical procedures for plaque removal, the endothelial cell layer of vessels is often lost. In this situation, a site-specific cell replacement strategy may be the optimal approach to restore vascular function and to prevent thrombosis and restenosis. In previous studies, even when the cells were locally applied by catheters, the restoration of blood flow resulted in the detachment of the majority of cells. Thus, the combination of MNPs and magnetic fields may be also a sophisticated strategy to permit an increased efficiency of cell replacement. In fact, the retention rate of MNP-loaded endothelial cells could be strongly enhanced by magnetized stents or vascular grafts in vivo [15-17]. To prevent the risks of vascular injury by stent positioning or end organ damage by interrupted blood flow, our group has established a strategy for an endothelial cell replacement with direct targeting of the vascular wall under flow conditions. Therefore, we first employed lentivirus/MNP complexes for the efficient concomitant transduction and MNP labeling of endothelial cells. These cells were then injected into the blood stream of a denuded murine carotid artery in vivo. In the initial study, we could site-specifically 
retain reporter gene expressing cells by a small magnet imposed on the carotid artery of the anesthetized animal [14]. To improve the efficiency of cell retention and to also modulate vascular function, in the next step, we optimized magnetic targeting and overexpressed the functional gene eNOS in the replacement cells. Using a specially designed magnet configuration [18] we obtained a radially symmetric deposition of endothelial cells at the inner surface of the vessel. Importantly, magnet-assisted overexpression of eNOS enabled enhanced NO production of the cells modulating vascular tone of murine vessels ex vivo and in vivo [19].

\section{Future perspective}

During the last years, the application of MNPs has provided major advances in experimental therapies of vascular diseases. The site-specific positioning of drugs, genetic vectors and cells in the vasculature using a combination of MNPs and magnetic fields increases their local therapeutic actions in the vascular wall and minimizes remote adverse effects. While for MNPbased drug and cell therapy strategies, functional improvements have been demonstrated in animal studies, experimental gene therapy is still awaiting evidence to profit from MNP-based techniques. In fact, the high number of recent clinical trials of vascular gene therapy with unsatisfactory results reveals the need for novel delivery concepts. However, also the translation of successful MNP-dependent targeting approaches to humans requires further efforts. In order to optimize MNPs, their composition needs to be improved enabling prolonged circulation in the blood stream while still supporting efficient targeting. To achieve this, our understanding of MNP surface modifications in the blood (e.g., the formation of a protein corona) needs to expand. Additionally, the uptake mechanisms by vascular cells but also the processes resulting in unintended MNP accumulation in liver and spleen require clarification. This would then permit the development of special surface modifications for efficient MNP shielding and the design of multimodal MNPs that are similarly suited for drug, gene and cell transfer as well as novel imaging approaches. These particles may also help to unravel so far unsolved questions about MNP distribution, fate, long-term effects and toxicity in the human body.

Apart from an improved MNP design, there is also a demand for more sophisticated magnetic fields, in

\section{References}

1 Wenzel D, Rieck S, Vosen S et al. Identification of magnetic nanoparticles for combined positioning and lentiviral transduction of endothelial cells. Pharm. Res. 29, 1242-1254 (2012). particular for human application. For MNP-based targeting approaches in large vessels with high blood flow velocities as found in humans, much stronger magnetic fields than in rodent models are required. Thus, to achieve an efficient radially symmetric retention of drugs, genetic vectors or cells in large or deep vessels, novel strategies comprising permanent or electromagnets with performance enhancing elements need to be developed. These magnet configurations then have to be explored in large animal models that better resemble the size and hemodynamics of human vessels. Additionally, for the application in humans innovative viral vector concepts with an optimized safety profile but also long-lasting expression are required. For cell therapy, autologous endothelial cells demonstrating a high regenerative potential, for example, endothelial progenitor cells or endothelial cells derived from induced pluripotent stem cells (iPS), would be ideal. Thus, novel protocols enabling the generation of high numbers of these cell types need to be established.

\section{"...the combination of MNPs and magnetic fields revealed the potential to open up new avenues for site-specific vascular therapies."}

In summary, the combination of MNPs and magnetic fields revealed the potential to open up new avenues for site-specific vascular therapies. Future studies will have to demonstrate functional vascular improvement for MNP-assisted gene therapy and the applicability in large animals and humans.

\section{Acknowledgements}

The author would like to thank the members of the DFG Research Unit FOR 917 "Nanoguide" for helpful discussions.

\section{Financial \& competing interests disclosure}

The work was supported by funding to the junior research group "Magnetic nanoparticles (MNPs) - endothelial cell replacement in injured vessels" by the Ministry of Innovation, Science, Research and Technology of the State of North RhineWestphalia. The author has no other relevant affiliations or financial involvement with any organization or entity with a financial interest in or financial conflict with the subject matter or materials discussed in the manuscript apart from those disclosed.

No writing assistance was utilized in the production of this manuscript. 
3 Schladt TD, Schneider K, Schild H, Tremel W. Synthesis and bio-functionalization of magnetic nanoparticles for medical diagnosis and treatment. Dalton Trans. 40, 6315-6343 (2011).

4 Kelkar SS, Reineke TM. Theranostics: combining imaging and therapy. Bioconjug. Chem. 22, 1879-1903 (2011).

5 Moulton KS, Heller E, Konerding MA, Flynn E, Palinski W, Folkman J. Angiogenesis inhibitors endostatin or TNP-470 reduce intimal neovascularization and plaque growth in apolipoprotein E-deficient mice. Circulation 99, 1726-1732 (1999).

6 Marx SO, Totary-Jain H, Marks AR. Vascular smooth muscle cell proliferation in restenosis. Circ. Cardiovasc. Interv. 4, 104-111 (2011).

7 Unverdorben M, Vallbracht C, Cremers B et al. Paclitaxelcoated balloon catheter versus paclitaxel-coated stent for the treatment of coronary in-stent restenosis. Circulation 119, 2986-2994 (2009).

8 Schiele TM, Krotz F, Klauss V. Vascular restenosis - striving for therapy. Expert Opin. Pharmacother. 5, 2221-2232 (2004).

9 Chorny M, Fishbein I, Yellen BB et al. Targeting stents with local delivery of paclitaxel-loaded magnetic nanoparticles using uniform fields. Proc. Natl Acad. Sci. USA 107, 8346-8351 (2010).

10 Winter PM, Neubauer AM, Caruthers SD et al. Endothelial alpha(v)beta3 integrin-targeted fumagillin nanoparticles inhibit angiogenesis in atherosclerosis. Arterioscler. Thromb. Vasc. Biol. 26, 2103-2109 (2006).

11 Cyrus T, Zhang H, Allen JS et al. Intramural delivery of rapamycin with alphavbeta3-targeted paramagnetic nanoparticles inhibits stenosis after balloon injury. Arterioscler. Thromb. Vasc. Biol. 28, 820-826 (2008).

12 George SJ, Baker AH. Gene transfer to the vasculature: historical perspective and implication for future research objectives. Mol. Biotechnol. 22, 153-164 (2002).

13 Chorny M, Fishbein I, Tengood JE, Adamo RF, Alferiev IS, Levy RJ. Site-specific gene delivery to stented arteries using magnetically guided zinc oleate-based nanoparticles loaded with adenoviral vectors. FASEB J. 27, 2198-2206 (2013).

14 Hofmann A, Wenzel D, Becher UM et al. Combined targeting of lentiviral vectors and positioning of transduced cells by magnetic nanoparticles. Proc. Natl Acad. Sci. USA 106, 44-49 (2009).

15 Pislaru SV, Harbuzariu A, Gulati R et al. Magnetically targeted endothelial cell localization in stented vessels. J. Am. Coll Cardiol. 48, 1839-1845 (2006).

16 Pislaru SV, Harbuzariu A, Agarwal G et al. Magnetic forces enable rapid endothelialization of synthetic vascular grafts. Circulation 114, I314-I318 (2006).

17 Polyak B, Fishbein I, Chorny M et al. High field gradient targeting of magnetic nanoparticle-loaded endothelial cells to the surfaces of steel stents. Proc Natl Acad. Sci. USA 105, 698-703 (2008).

18 Heidsieck A, Vosen S, Zimmermann K, Wenzel D, Gleich B. Analysis of trajectories for targeting of magnetic nanoparticles in blood vessels. Mol. Pharm. 9, 2029-2038 (2012).

19 Vosen S, Rieck S, Heidsieck A et al. Vascular repair by circumferential cell therapy using magnetic nanoparticles and tailored magnets. ACS Nano 10, 369-376 (2016). 